\title{
SPHINX : générateur de rayons $X$ pulsés, ultra-compact à cadence élevée
}

R. Viladrosa, A. Khacef, C. Cachoncinlle et J.M. Pouvesle

GREMI, CNRS, Université d'Orléans, BP. 6759, 45067 Orléans cedex 2, France

\begin{abstract}
Résumé : Des progrès significatifs ont été réalisés ces dernières années dans le développement des sources $X$ impulsionnelles, réglables en énergie rayonnée, ultra-compactes et réellement portables. En utilisant une technique de lignes adaptées et de nouveaux matériaux nous avons obtenu des doses à $1 \mathrm{~m}$ qui conduisent dès maintenant à des applications intéressantes aussi bien en recherche, en médecine, qu'en industrie.
\end{abstract}

\section{INTRODUCTION}

Disposer facilement d'une source de rayonnement $\mathrm{X}$ impulsionnelle, récurrente et suffisamment intense n'est pas trivial. La plupart des dispositifs sont de grandes dimensions et difficilement transportables, ou sont limités par leur très faible taux de répétition et ne correspondent pas aux besoins de nombreuses applications industrielles ou scientifiques.

Nous proposons une nouvelle source qui trouvera sa place là ou disponibilité et compacité sont primordiales, sans négliger pour autant les énergies mises en jeu.

\section{LE GENERATEUR}

\subsection{Présentation générale}

Le générateur SPHINX (Source de PHotons Impulsionnelle Nanoseconde $X$ ) très compact, est placé dans une petite malle métallique pour être transporté . L'intégration dans cet ensemble continûment métallique, du groupe de puissance à la diode $X$ déportée, lui confère la compatibilté électromagnétique (CEM) nécessaire à son environnement.

Le volume réduit $\left(0.1 \mathrm{~m}^{3}\right)$ et le poids ( $45 \mathrm{~kg}$ ) de l'appareil, comprenant le système de pompage et l'alimentation THT en font un instrument aisément transportable sur site ou dans un laboratoire. Il est conçu pour être posé sur une table et connecté simplement sur une prise $220 \mathrm{~V} / 16 \mathrm{~A}$. Le refroidissement est assuré par une ventilation d'air forcé.

\subsection{Caractéristiques physiques}

Le diode $\mathrm{X}$, fonctionnant aussi bien sous vide résiduel d'air que sous faible pression de gaz, est à même d'émettre des impulsions de photons $X$ d'une durée de quelques nanosecondes, et cela à cadence élevée. Cette diode, en verre, est aisément démontable, ce qui facilite le nettoyage ou le remplacement des électrodes. Un dispositif mécanique 
permet le réglage de la distance inter-électrodes pour compenser l'usure de celles-ci ou optimiser les conditions de décharge. La diode est fixée à l'extrémité d'un câble souple, dont la longueur peut atteindre plusieurs mètres, afin d'aider sa manipulation et son positionnement. Pour une meilleure sûreté, elle est gainée par un tube en acier qui assure la protection $\mathrm{X}$ en dehors de la direction principale d'émission.

La tension de charge, réglable de $10 \mathrm{kV}$ à $30 \mathrm{kV}$, fournie par un chargeur de condensateurs est commutée par l'intermédiaire d'un éclateur déclenché. Une partie de l'énergie est transférée jusqu'à la diode $\mathrm{X}$ par les lignes de mise en forme permettant la multiplication de tension (jusqu'à $\times 8$ ). Du fait de cette conception originale, les énergies comme les tensions générées à la diode sont adaptables en fonction des besoins.

Le boîtier de télécommande sur lequel sont effectués les réglages et affichages des tensions et fréquences de décharge est relié à l'ensemble de puissance par des fibres optiques $(10 \mathrm{~m})$.

\subsection{Caractéristiques du faisceau $\mathbf{X}$}

Dans sa configuration actuelle le générateur fonctionne en mode cadencé du régime monocoups jusqu'à $50 \mathrm{~Hz}$. Pour une énergie maximum stockée de 7 joules, des doses de $1 \mathrm{mR}^{(1)}$ par tir à 1 mètre ont été obtenues avec des photons $\mathrm{X}$ d'énergie comprise entre 5 et $150 \mathrm{keV}$. La durée des impulsions variant entre 20 et 30 ns.

\section{CONCLUSION}

Le champ d'application de ce système intégré, et aisément transportable, est relativement large, autant pour des applications industrielles sur site, que scientifiques. Parmi ces celles-ci, la ciné-radiographie médicale, la visualisation de phénomènes ultra-rapides ou balistiques représentent quelques développements intéressants de ce générateur.

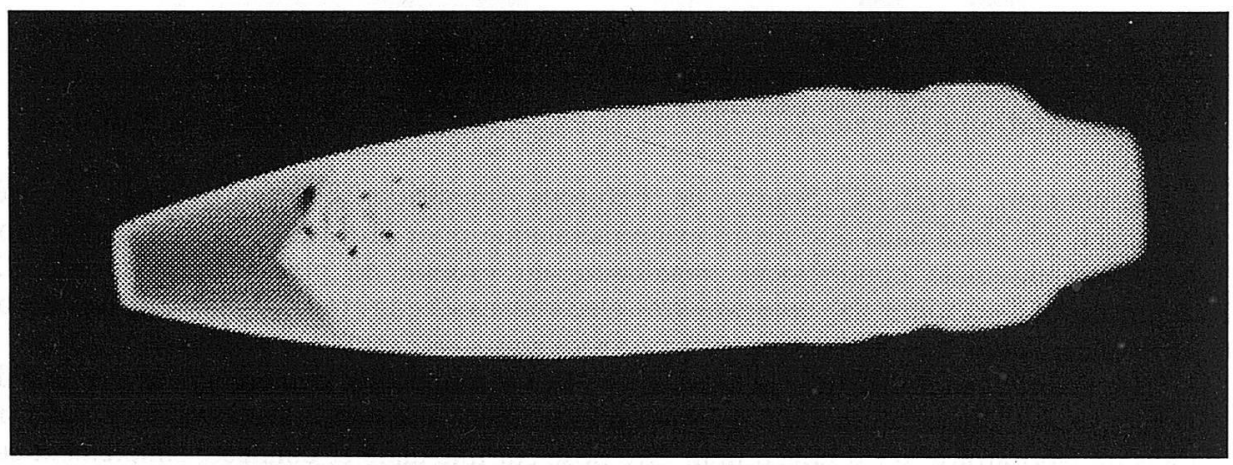

Figure 1: Radiographie obtenue avec le SHINX, d'un projectile de moyen calibre à $700 \mathrm{~m} / \mathrm{s}$, à $20 \mathrm{~m}$ de la bouche. (autorisation reproduction DGA - ETBS).

\section{Remerciements.}

Les auteurs remercient A.Quilgard (LCSR, Orléans) pour son aide.

(1) $1 \mathrm{R}=1$ Roentgen $=2.4810^{-4} \mathrm{C} \mathrm{kg}^{-1}$ 